\title{
ANÁLISE DE RESÍDUOS DE PESTICIDAS EM TOMATES POR CROMATOGRAFIA EM CAMADA DELGADA
}

\author{
Solange Leite Moraes e Maria Olímpia Oliveira Rezende \\ Instituto de Química de São Carlos, Universidade de São Paulo, CP 780, 13560-970 São Carlos - SP \\ Lia Emi Nakagawa e Luiz Carlos Luchini \\ Instituto Biológico de São Paulo, Av. Cons. Rodrigues Alves, 1252, São Paulo - SP
}

Recebido em 31/10/00; aceito em 6/9/01

\begin{abstract}
ANALYSIS OF RESIDUE PESTICIDE IN TOMATOES BY THIN LAYER CHROMATOGRAPHY. Pesticide residues are determined by thin layer chromatography (TLC) using the Hill reaction as a detection method. Tomatoes samples without pesticide were fortified with atrazine, diuron, chloroxuron and metribuzin, and were applyed in silica gel plates with the help of a microsyringe. The pesticides were elued with ethyl acetate. There was no need of cleaning up because no interference was noticed. After the revelation of the plates, the diameters of the spots were measure by using a rule. The range of the determined concentration for all the pesticides was from 0.1 to1.0 $\mathrm{ng} / \mu \mathrm{L}$. The results obtained through TLC can be used for semi-quantitative analysis. The results obtained were compared to gas and liquid chromatography, showing good agreement between both techniques.
\end{abstract}

Keywords: tomatoes; Hill reaction; thin layer chromatography.

\section{INTRODUÇÃO}

Devido aos riscos que os resíduos de pesticidas trazem à saúde de quem consome alimentos contaminados com eles, há necessidade de um monitoramento constante dos níveis de resíduos dos mesmos nos mais variados produtos ${ }^{1}$.

Instituições com laboratórios capazes de analisar resíduos de pesticidas são escassas em muitas regiões e totalmente ausentes em outras. Isto porque os métodos analíticos usualmente empregados nestas análises, que são os cromatográficos, requerem equipamentos sofisticados e pessoal técnico especializado. Além disso, essas técnicas demandam dos laboratórios de resíduos grande investimento em infra-estrutura e materiais nem sempre acessíveis ${ }^{2}$.

Assim, devido ao alto custo das análises, necessidade de pessoal especializado e sofisticação dos métodos empregados, a elevação do número de amostras a serem analisadas não pode ser feita na justa medida da demanda. Conseqüentemente, a busca de novos métodos para monitorar resíduos de pesticidas deve ser estimulada tanto sob o ponto de vista ambiental como o de saúde pública ${ }^{3,4}$. Desta forma, neste trabalho pretendeu-se desenvolver uma metodologia simples e de baixo custo, sem a necessidade de equipamentos sofisticados e de fácil aplicação local, isto é, nas próprias regiões produtoras e distribuidoras de frutas e legumes por exemplo, podendo-se, assim, ampliar o monitoramento e controlar a qualidade desses produtos nas principais regiões agrícolas do Brasil.

A cromatografia em camada delgada (TLC) foi a metodologia escolhida e desenvolvida neste estudo para a determinação dos resíduos. As vantagens desse método são a simplicidade, a instrumentação de baixo custo e a versatilidade, apresentando-se, portanto, adequado à execução de determinações em série. Além disso, a TLC pode ser usada quando as possibilidades de aplicação de outros métodos são limitadas pelas propriedades da amostra, como por exemplo, substâncias termolábeis, baixa volatilidade ou presença de quantidades consideráveis de impurezas dificultando a análise (resinas, pigmentos, etc. $)^{5-7}$. O sucesso da separação cromatográfica depende do

\footnotetext{
*e-mail: mrezende@iqsc.sc.usp.br
}

método de detecção. Substâncias coloridas são, é claro, visíveis como manchas distintas ao fim da corrida. Uma das maiores vantagens da TLC é a grande variedade de reagentes de detecção usados, os quais podem ser mais ou menos seletivos. Quando o reagente dissolvido no solvente adequado é aplicado na placa, produz-se uma mancha colorida in situ. O reagente pode ser classificado como não específico se produzir manchas coloridas com uma variedade de compostos, ou específico se for somente reativo com compostos contendo um grupo funcional particular ${ }^{5,8}$.

O método mais comum para análises quantitativas em TLC utiliza densitômetros contudo, existem vários outros métodos de detecção muito sensíveis que também podem ser utilizados com esta finalidade como, por exemplo, a reação de Hill. Esta reação faz parte do processo de fotossíntese das plantas. Cerca de $40 \%$ dos herbicidas (uracilas, feniluréias e triazinas, dentre outros) destroem as ervas daninhas devido à capacidade que possuem em interferir com o processo fotossintético.

A reação $2 \mathrm{H}_{2} \mathrm{O}+2 \mathrm{~A} \rightarrow 2 \mathrm{AH}_{2}+\mathrm{O}$ é conhecida como reação de Hill. No esquema, A representa um aceptor artificial de hidrogênio, em sua forma oxidada e $\mathrm{AH}_{2}$ representa um aceptor artificial de hidrogênio, em sua forma reduzida. $\mathrm{O}$ aceptor utilizado por Hill foi o 2,6-diclorofenolindolfenol, um corante não biológico que na sua forma oxidada (A) é azul e na forma reduzida $\left(\mathrm{AH}_{2}\right)$ é incolor ${ }^{9}$.

Além desse processo, existem outros processos químicos e biológicos que são responsáveis pela detecção de resíduos de pesticidas em TLC e a maior ou menor sensibilidade e seletividade desses compostos vão depender da capacidade que os mesmos possuem de inibir determinadas reações químicas ou biológicas ${ }^{10}$. A literatura apresenta vários exemplos desses métodos capazes de detectar pesticidas na ordem de nanogramas ${ }^{11-21}$. Compostos organoclorados, fenoxiácidos e triazinas podem ser detectados com nitrato de prata ${ }^{16}$. Inseticidas organofosforados e carbamatos podem ser detectados por reações enzimáticas ${ }^{15,19}$. Herbicidas triazínicos e derivados de uréia, por inibição da fotossíntese ${ }^{21}$.

Similarmente às outras técnicas cromatográficas, a identificação das substâncias é também contestável em TLC, já que compostos diferentes podem apresentar o mesmo valor de Rf. Além disso, são necessários padrões puros para identificar compostos desconheci- 
dos. Teoricamente, seriam necessários reagentes específicos para cada identificação. O uso combinado de vários reagentes de detecção ajuda na identificação de tipos ou classes de compostos, mas não na identificação do composto individual. A única solução para a identificação é o uso combinado de técnicas ${ }^{5-7}$.

A tomaticultura, apesar de ser uma cultura rentável, costuma atravessar fases críticas devido à ocorrência freqüente e constante de pragas durante todo o ciclo da planta. Face à rápida proliferação de pragas, os agricultores realizam aplicações preventivas de pesticidas até duas vezes por semana, o que além de aumentar o custo da produção pode induzir resistência às pragas ${ }^{22,23}$.

Este estudo foi realizado com o intuito de investigar a possibilidade de aplicação do método de cromatografia em camada delgada (TLC) em combinação com métodos de extração e "clean-up" para análise de resíduos de pesticidas em amostras de tomate, devido aos riscos que os resíduos de pesticidas trazem à saúde de quem os consome e frente à necessidade de um monitoramento constante dos níveis de resíduos.

\section{PARTE EXPERIMENTAL}

\section{Reagentes e Soluções}

Todos os padrões de pesticidas, atrazina, cloroxuron, diuron e metribuzin, foram fornecidos pela Agência Internacional de Energia Atômica (IAEA) com pureza superior a 99\%. As soluções estoque, na concentração de $1 \mathrm{mg} / \mathrm{mL}$ cada, foram preparadas em acetona e as soluções trabalho foram preparadas por diluição da solução estoque. Bio-beads SX3 (200-400 mesh) foram usadas como gel para cromatografia de permeação em gel (GPC). O solvente acetato de etila e as placas de sílica gel $60 \mathrm{~F}$ de $(20 \times 20) \mathrm{cm}^{2}$ foram adquiridas da Merck. A Figura 1 apresenta os herbicidas estudados.

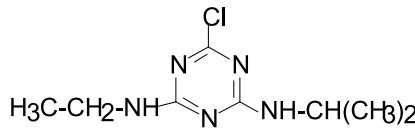<smiles>CN(C)C(=O)Nc1ccc(Oc2ccc(Cl)cc2)cc1</smiles>

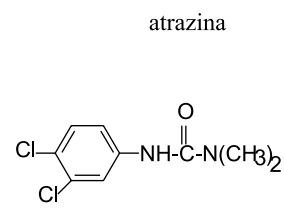

diuron<smiles>Cc1nnc(C(C)(C)C)c(=O)n1N</smiles>

metribuzin
Figura 1. Estruturas dos herbicidas estudados ${ }^{24}$

As placas cromatográficas foram ativadas a $105{ }^{\circ} \mathrm{C}$ durante 1 hora. As cubas foram saturadas durante 30 minutos, antes de sua utilização. Para a aplicação dos padrões de pesticidas utilizou-se microsseringa de $25 \mu \mathrm{L}$.

A quantidade mínima detectada (MDQ) foi determinada diminuindo-se gradativamente a quantidade do composto aplicada até se obter a menor quantidade claramente visível como mancha na placa.

Os valores de $\mathrm{Rf}$ foram determinados em cubas de desenvolvimento mantidas em salas a $20^{\circ} \mathrm{C}$. O eluente ficou em equilíbrio com a fase vapor pela inserção de um papel de filtro na cuba de desenvolvimento, durante 30 minutos. Após o desenvolvimento das placas, estas foram secas e reveladas.

A Figura 2 ilustra como foram aplicadas as soluções de pesticidas para determinação em TLC.
$20 \mathrm{~cm}$

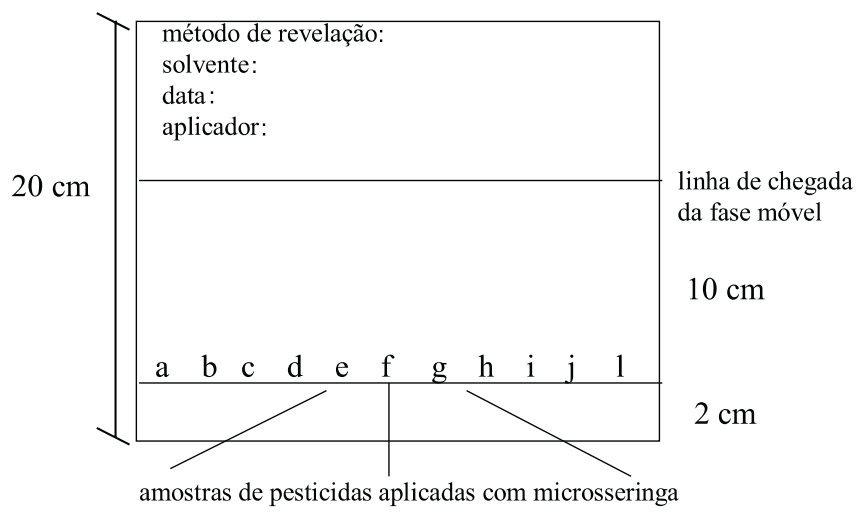

Figura 2. Ilustração da placa cromatográfica

\section{Método de detecção}

Inibição da fotossíntese [reação de Hill] ${ }^{3}$

O método de detecção utilizado neste trabalho foi o método de inibição da fotossíntese. Este método é específico para compostos que atuam na inibição da fotossíntese.

Plantaram-se sementes de trigo em potes que foram colocados próximos à janela. Em solos de boa qualidade, as folhas de trigo podem ser colhidas em duas semanas. Após esse período, 30g de folhas de trigo foram cortadas e colocadas em um cadinho de porcelana, acrescentando-se $3 \mathrm{~mL}$ de glicerina, $15 \mathrm{~mL}$ de água destilada e $5 \mathrm{~g}$ de areia. A mistura, após macerada até homogeneidade, foi filtrada em um béquer em quatro camadas de gaze. Deve-se cobrir o béquer contendo o extrato das folhas com papel alumínio e mantê-lo em geladeira até o momento da determinação, que não deve ultrapassar 12 horas.

A solução tampão bórax foi preparada pela adição de $350 \mathrm{~mL}$ de solução $0,05 \mathrm{~mol} / \mathrm{L}$ de bórax a $150 \mathrm{~mL}$ de $\mathrm{HCl} 0,1 \mathrm{~mol} / \mathrm{L}$. A solução de 2,6 - diclorofenolindolfenol de sódio (DCPIP) foi preparada misturando-se $200 \mathrm{mg}$ de 2,6 - diclorofenolindolfenol de sódio a 500 $\mathrm{mL}$ de tampão bórax.

A solução reagente foi preparada adicionando-se $20 \mathrm{~mL}$ do extrato das folhas a $10 \mathrm{~mL}$ da solução de DCPIP.

A revelação foi feita borrifando-se a placa com a solução reagente e expondo-a à lâmpada de $60 \mathrm{~W}$ a uma distância de $20 \mathrm{~cm}$. Em aproximadamente 3 minutos manchas azuis apareceram na placa de fundo esverdeado.

\section{Determinação das curvas analíticas em TLC}

Para a elaboração das curvas analíticas por TLC, utilizaram-se 5 concentrações padrão (MDQ, 2xMDQ, 4xMDQ, 6xMDQ e 8xMDQ, para cada pesticida), em acetona. As soluções padrão foram aplicadas entre 5-25 $\mu \mathrm{L}$, com manchas que produziram entre 3-4 mm de diâmetro.

Após o desenvolvimento da placa, mediram-se os diâmetros (1 e 2) da mancha e calculou-se a média, conforme ilustra a Figura 3.

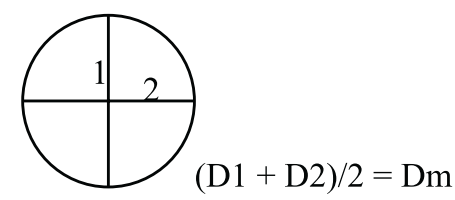

Figura 3. Medida do diâmetro da mancha 
Todas as medidas foram realizadas em triplicata. Construíram-se gráficos com a média dos diâmetros, Dm, em mm, em função da quantidade do composto aplicada (ng).

\section{Determinação das curvas analíticas em GC e HPLC}

Para a determinação das curvas analíticas em GC e HPLC novas soluções padrão foram preparadas em metanol na concentração de $100 \mathrm{mg} / \mathrm{L}$. A partir dessa solução estoque fizeram-se as diluições nas mesmas concentrações utilizadas na determinação das curvas analíticas em TLC.

Para a determinação cromatográfica dos compostos atrazina, diuron, cloroxuron e metribuzin utilizou-se um cromatógrafo a gás com detector de captura de elétrons e injetor split, Hewlett Packard 5890, série II, operando sob as seguintes condições: coluna Ultra 2 (5\% fenil metil siliconada) $25 \mathrm{~m} \mathrm{x} \mathrm{0,2} \mathrm{mm} \times 0,33 \mu \mathrm{m}$; temperatura do forno $\left(180{ }^{\circ} \mathrm{C}\right)$; temperatura do injetor $\left(270{ }^{\circ} \mathrm{C}\right)$; temperatura do detector $\left(320{ }^{\circ} \mathrm{C}\right)$; volume de injeção $1 \mu \mathrm{L}$; modo split (1: 13); gás de arraste hidrogênio $1 \mathrm{~mL} \mathrm{~min}{ }^{-1}$; gás auxiliar nitrogênio $1 \mathrm{~mL} \mathrm{~min}{ }^{-1}$; com programação da rampa de temperatura sendo temperatura inicial de $180{ }^{\circ} \mathrm{C}$ por $2,5 \mathrm{~min}$, seguida do incremento de $3{ }^{\circ} \mathrm{C} / \mathrm{min}$ por 1 min e de $10{ }^{\circ} \mathrm{C} / \mathrm{min}$ até $280{ }^{\circ} \mathrm{C}$.

As determinações cromatográficas para o herbicida cloroxuron foram feitas utilizando um cromatógrafo líquido SHIMADZU com bombas LS-10AD acoplado a um detector SPD-M6A com arranjo de diodos, UV-Vis. Utilizou-se uma coluna: C-18, com $30 \mathrm{~cm}$ de comprimento e $4 \mathrm{~mm}$ de diâmetro interno. As condições analíticas foram: água/metanol (20-80 v/v) como eluente, razão de fluxo $1 \mathrm{~mL} / \mathrm{min}$; volume de injeção: $20 \mu \mathrm{L}$ ("loop”) e detecção em 254 nm.

\section{Condições cromatográficas para o "clean-up"}

O processo de "clean-up"utilizado neste estudo foi a cromatografia de permeação em gel (GPC), sistema semi-automático KLSX-3, operando com pressão de nitrogênio de 0,4 bar. Utilizou-se uma coluna de vidro, $10 \mathrm{~mm}$ id x $20 \mathrm{~cm}$, empacotada com Bio-Beads SX-3, 200-400 mesh. O sistema de eluição foi acetato de etila/ cicloexano (1:1 v/v); utilizando-se nitrogênio como gás de arraste. A razão do fluxo foi de $1 \mathrm{~mL} / \mathrm{min}$.

O gel utilizado para preencher a coluna foi misturado sob agitação com acetato de etila e cicloexano $(1: 1 \mathrm{v} / \mathrm{v})$ durante 5 horas em mesa agitadora.

Primeiramente, realizou-se a calibração da coluna de permeação em gel com o intuito de descobrir quais eram as frações em que os pesticidas estudados e o pigmento natural do tomate eluíam.

\section{Definição das frações de eluição do pigmento}

Amostras de tomates (em triplicata) isentas de pesticidas foram injetadas em GPC, após o processo de extração. Injetou-se 0,5 mL do extrato (branco) e coletaram-se 30 frações de $1 \mathrm{~mL}$ cada. As frações coletadas foram determinadas em um espectrofotômetro de absorção HITACHI, modelo U-1100, operando em 470 nm, comprimento de onda em que ocorre o máximo de absorção para o pigmento vermelho do tomate.

\section{Definição das frações de eluição para os pesticidas}

Os extratos de tomates (testemunhas) foram fortificados com pesticidas radiomarcados na concentração de 180.000 dpm e, após o processo de extração, foram injetadas em GPC. Injetou-se $0,5 \mathrm{~mL}$ do extrato e coletaram-se 30 frações de $1 \mathrm{~mL}$ cada. As frações coletadas foram analisadas em um espectrofotômetro de cintilação líquida, após a adição de $7 \mathrm{~mL}$ da solução de $0,4 \mathrm{~g}$ de POP (2,5 difeniloxazole), 0,2g de POPOP (1,4 bis 2-(5-difeniloxazole)-benzeno) e $1340 \mathrm{~mL}$ de tolueno e $660 \mathrm{~mL}$ de renex ${ }^{25}$. Os pesticidas radiomarcados foram: ${ }^{14} \mathrm{C}$ atrazina e ${ }^{14} \mathrm{C}$-diuron, representando as classes dos pesticidas s-triazinas e uréia, respectivamente. Determinou-se a recuperação dos pesticidas após a passagem pela coluna cromatográfica.

\section{Processo de Extração e recuperação dos Pesticidas}

Os tomates foram adquiridos em lojas de produtos orgânicos, denominação esta dada aos alimentos supostamente sem pesticidas. Esses tomates foram analisados de acordo com a metodologia aqui desenvolvida, não sendo constatada a presença de pesticidas neles.

As amostras de tomate foram extraídas segundo o procedimento esquematizado na Figura 4. A quantidade de pesticida adicionada em cada amostra foi 1,0 $\mu \mathrm{g}$ de atrazina, 1,0 $\mu \mathrm{g}$ cloroxuron, $1,0 \mu \mathrm{g}$ diuron e $0,50 \mu \mathrm{g}$ de metribuzin, considerando $60 \mathrm{~g}$ de amostra. Após a extração, as amostras foram submetidas a um processo de "cleanup" em cromatografia de permeação em gel, para remoção dos interferentes e os pesticidas adicionados foram determinados por TLC, com o objetivo de avaliar a recuperação do método.

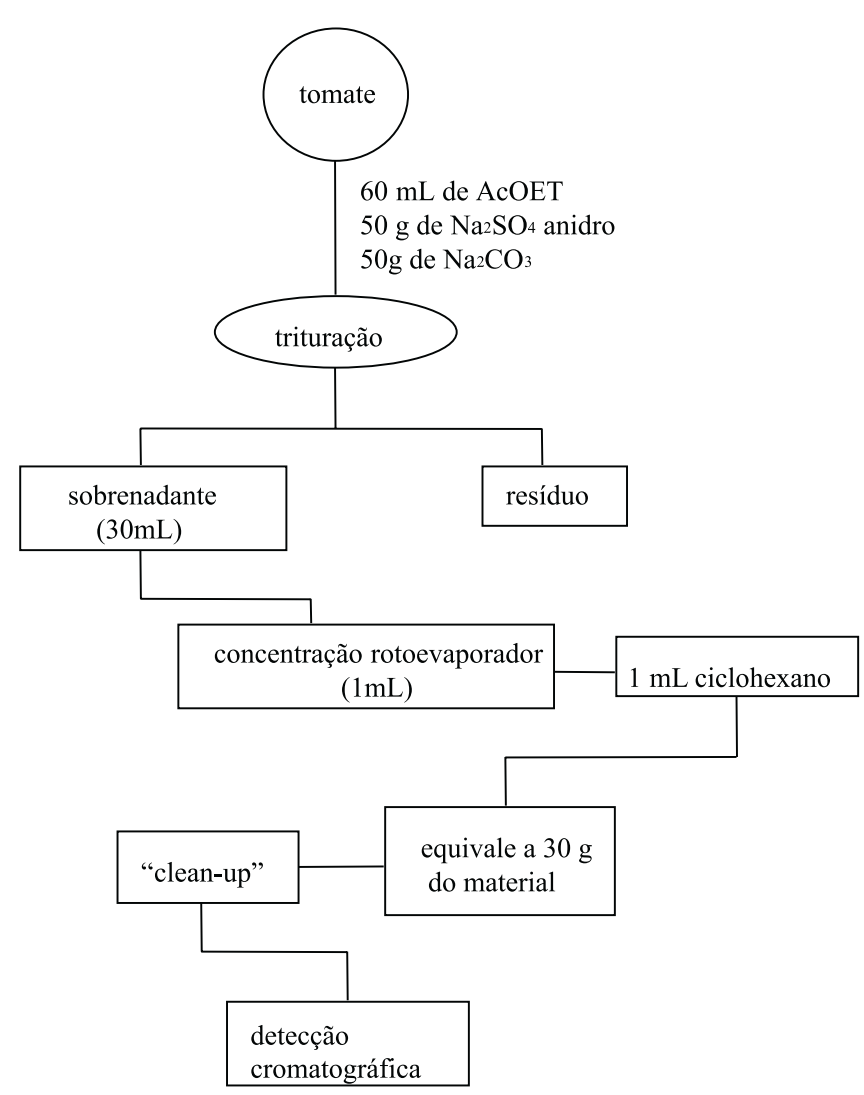

Figura 4. Diagrama do procedimento utilizado na análise de pesticidas em amostras de tomates

Injetou-se 0,5 mL do extrato fortificado em GPC e as frações correspondentes ao pigmento foram descartadas. As demais frações de 14 a 30 foram coletadas, concentradas em rotaevaporador e ressuspendidas em $0,5 \mathrm{~mL}$ de acetona para aplicação nas placas de TLC. As frações contendo os pesticidas foram determinadas anteriormente por procedimento semelhante usando-se apenas pesticidas radiomarcados. As mesmas amostras (após mudança de solvente para metanol) foram determinadas por cromatografia gasosa (GC) e por 
cromatografia líquida de alta eficiência (HPLC) para confirmação dos resultados de recuperação.

\section{RESULTADOS E DISCUSSÃO}

Todos os padrões de herbicidas utilizados neste trabalho foram testados usando o método de detecção reação de Hill.

Após a revelação da placa as cores dos pesticidas são nitidamente visíveis. As cores obtidas para cada pesticida dependem do método de detecção utilizado. Para o método de detecção reação de Hill as manchas apresentaram colorido azul.

ORRf ${ }_{\text {atrazina }}$ é definido como o Rf do pesticida em relação ao pesticida atrazina. É medido através do Rf pesticida/Rf atrazina sendo ambos aplicados na mesma placa, de forma que os possíveis interferentes sejam compensados. Assim, os valores de Rf de todos os pesticidas são calculados em relação a um único parâmetro: o Rf da atrazina. Os valores de $\mathrm{RRf}_{\text {atrazina }}$ foram determinados segundo a equação:

$\mathrm{RRf}_{\text {atrazina }}=\frac{\text { média Rf pesticida }}{\text { média Rf atrazina }}$

Na Tabela 1 apresentam-se os valores de Rf, os valores de Rf com relação à atrazina $\left(\mathrm{RRf}_{\text {atrazina }}\right)$ para o sistema de eluição acetato de etila e a MDQ. Com base nos resultados, os valores de RRf são mais precisos para identificação do composto do que os valores de Rf, uma vez que os valores de Rf divergem muito de um laboratório para outro. A MDQ de um pesticida é definida como sendo a quantidade do padrão analítico, expressa em nanogramas, que produz manchas claramente visíveis na placa ${ }^{3}$. Portanto, quanto menores os valores da MDQ, maior é a sensibilidade do método para o pesticida.

Tabela 1. Detectabilidade dos pesticidas pelo método de detecção reação de Hill

\begin{tabular}{lllll}
\hline \multirow{2}{*}{ herbicidas } & \multicolumn{1}{c}{ Rf } & \multicolumn{2}{c}{ RRf } & \\
& AcOEt & \multicolumn{2}{c}{ AcOEt } & MDQ(ng) \\
& & Exp. & Liter. $^{3}$ & \\
\hline atrazina & $0,65 \pm 0,01$ & 1,0 & 1,0 & 0,7 \\
cloroxuron & $0,45 \pm 0,01$ & 0,69 & 0,57 & 0,5 \\
diuron & $0,53 \pm 0,0$ & 0,81 & 0,60 & 0,5 \\
metribuzin & $0,69 \pm 0,01$ & 1,06 & 1,03 & 0,05 \\
\hline
\end{tabular}

MDQ = quantidade mínima detectada

Como o método de detecção reação de Hill é um método específico, para compostos que podem atuar na inibição da fotossíntese, todos os herbicidas utilizados neste trabalho foram detectados, uma vez que os mesmos podem atuar no processo fotossintético.

\section{Determinação das Curvas Analíticas em TLC}

Para a determinação das curvas analíticas, as medidas dos diâmetros da mancha foram feitas utilizando-se régua. Deve-se, portanto, levar em consideração os erros das medidas tais como, imprecisão da régua, vizualização da mancha, nitidez de contorno, etc.

A Figura 5 mostra as curvas analíticas obtidas para cada pesticida no sistema de eluição acetato de etila, método reação de Hill. O diâmetro da mancha representa a média de triplicatas.

\section{Testes da Eficiência do "Clean-up” em Coluna de Permeação em Gel}

Para avaliar qual era a fração de eluição do pigmento e dos pesticidas foram determinadas as absorções das diferentes frações
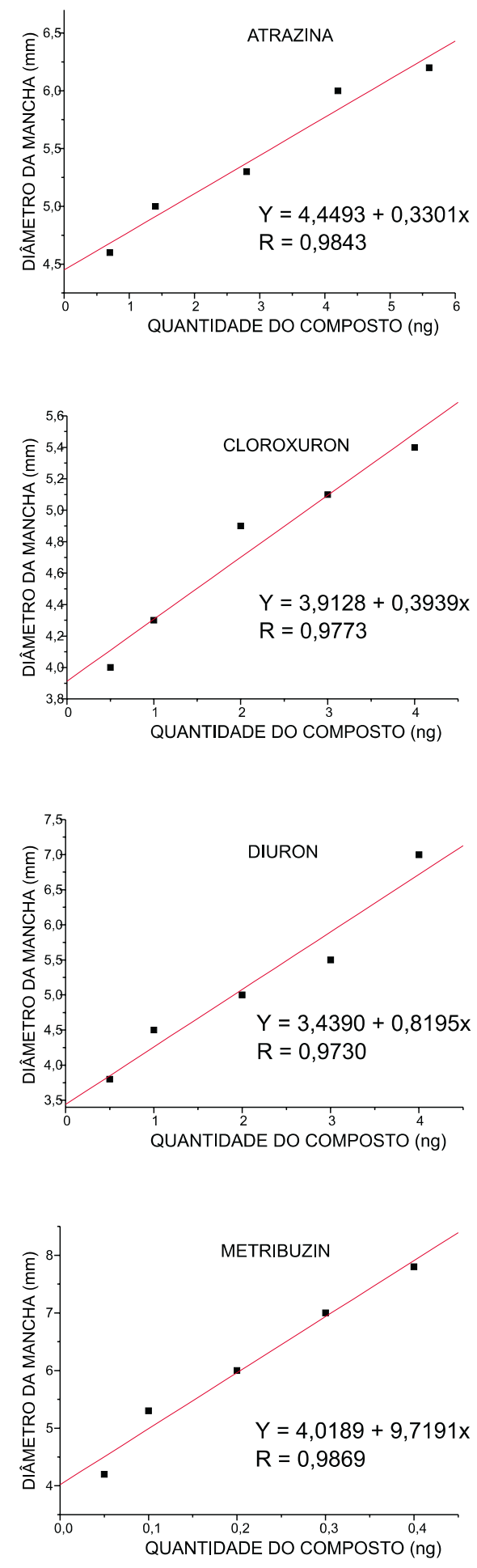

Figura 5. Curvas analíticas para o sistema de eluição acetato de etila, método reação de Hill

coletadas para amostras de tomate isentas de pesticidas (branco). $\mathrm{Na}$ Figura 6 apresenta-se a absorção do extrato de tomate nas diferentes frações coletadas.

Pelas figuras verifica-se que as frações nas quais ocorre a eluição do pigmento são as frações de número 9 a 13, ou seja, nessas frações houve maior absorção do pigmento. Esta maior absorção pode ser visualmente observada durante as coletas das frações devido à coloração vermelha do extrato de tomate. 


\section{Extrato de tomate \\ (branco) na coluna do GPC}

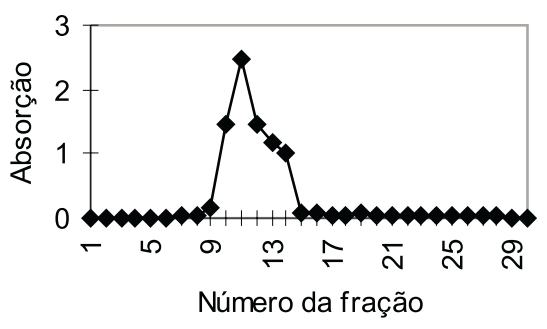

\section{Extrato de tomate (branco) na coluna de GPC}

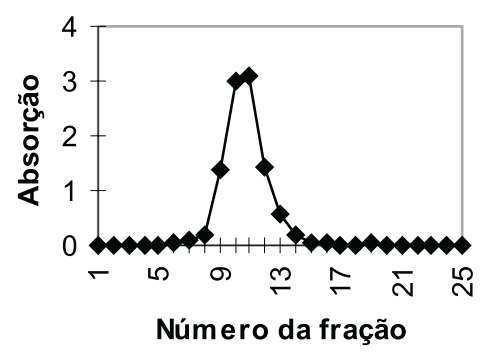

\section{Extrato de tomate (branco) coluna do GPC}

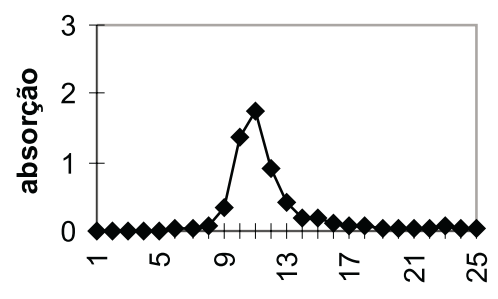

Número da fração

Figura 6. Curva de absorção de diferentes extratos de tomates (branco) nas diferentes frações $(\lambda=470 \mathrm{~nm})$

Para avaliar as frações de eluição dos pesticidas, as diferentes frações coletadas foram determinadas em um espectrofotômetro de cintilação líquida. Utilizaram-se pesticidas radiomarcados de diferentes classes químicas. Na Figura 7 apresentam-se as frações nas quais os pesticidas eluem.

Pela Figura 7 verifica-se que a eluição do pesticida diuron, pertencente à classe uréia, ocorre nas frações 13 a 19, enquanto que o pesticida atrazina, pertencente à classe das s-triazinas, co-elui com o pigmento de tomate nas frações entre 11 e 18 . O processo de "clean-up" para o herbicida atrazina não produz boa eficiência de separação. Pela Figura 8 visualizamos a não separação do pigmento juntamente com o herbicida, após passagem pela coluna de permeação em gel.

Teste de Recuperação dos Pesticidas Radiomarcados

Os extratos de tomates foram fortificados com os pesticidas radiomarcados. A Tabela 2 apresenta os valores de recuperação dos pesticidas após a passagem pela coluna do GPC.

\section{Pesticida na coluna de GPC}
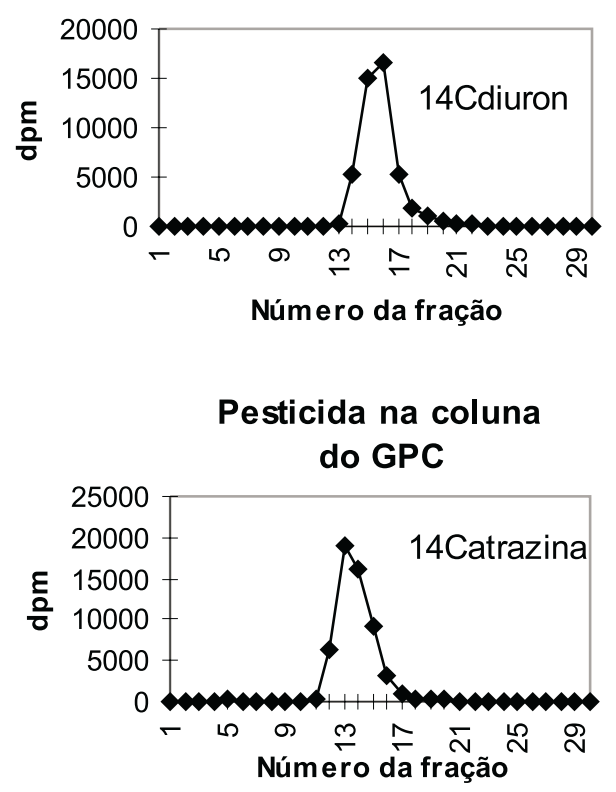

Figura 7. Separação das classes de pesticidas nas diferentes frações

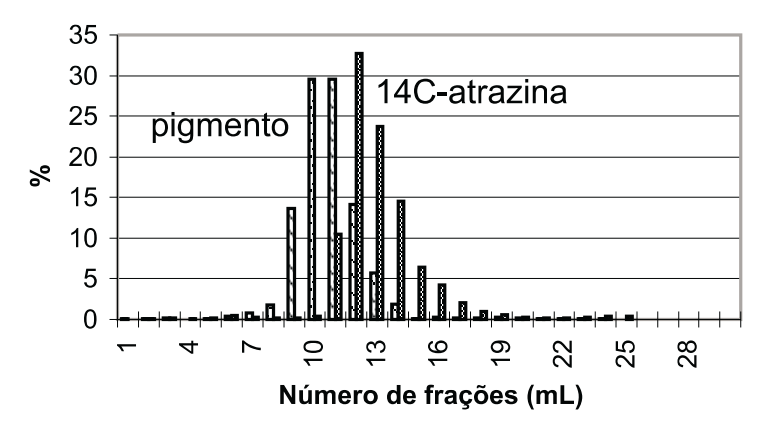

(a)

\section{Representação da separação $14 C$ atrazina + pigmento}

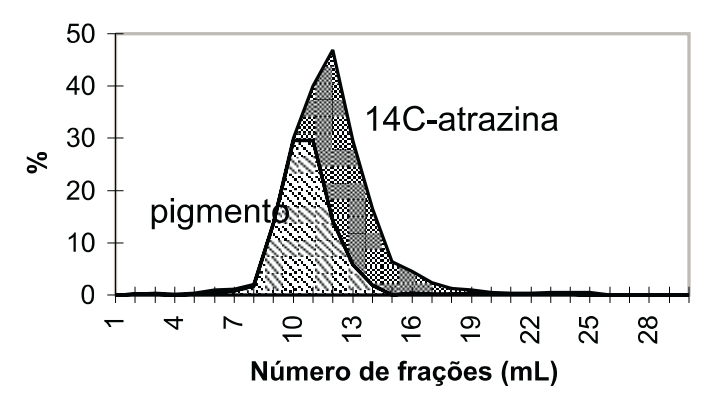

(b)

Figura 8. Separação do pigmento e do pesticida ${ }^{14} \mathrm{C}$-atrazina na coluna do GPC (a); visualização do pesticida e pigmento na coluna do GPC (b) 
Tabela 2. Recuperação dos padrões de pesticidas radiomarcados após "clean-up" por GPC

\begin{tabular}{lcc}
\hline Pesticida & Fortificação $(\mathrm{dpm})$ & Recuperação $(\%)$ \\
\hline${ }^{14}$ C-atrazina & 180.000 & $80,0 \pm 0,5$ \\
${ }^{14}$ C-diuron & 180.000 & $87,0 \pm 0,8$ \\
\hline
\end{tabular}

Pelos valores apresentados na Tabela 2 nota-se a eficiência da coluna no processo de "clean-up".

\section{Estudo Cromatográfico em GC-ECD e HPLC-UV}

Para a determinação dos tempos de retenção, determinou-se cada composto individualmente, depois fez-se uma mistura de todos os compostos sensíveis ao detector de captura de elétrons e traçou-se a curva analítica.

Testou-se o pesticida cloroxuron nas condições descritas mas não se obteve resposta para esse composto. $\mathrm{O}$ composto cloroxuron foi determinado por cromatografia líquida de alta eficiência (HPLC).

A Tabela 3 mostra os valores obtidos para elaboração das curvas analíticas e os tempos de retenção obtidos através de um GC-ECD e HPLC.

Tabela 3. Curvas analíticas dos padrões de pesticidas obtidas por GC e HPLC

\begin{tabular}{lcccc}
\hline Pesticida & $\operatorname{tr}(\min )$. & Detecção & E. Q. & $\mathrm{R}$ \\
\hline atrazina & 6,7 & ECD & $\mathrm{Y}=7086,6+9662,8 \mathrm{x}$ & 0,9988 \\
cloroxuron & 8,9 & HPLC & $\mathrm{Y}=21797,7+5656,3 \mathrm{x}$ & 0,9990 \\
diuron & 2,4 & ECD & $\mathrm{Y}=6243,6+12766,6 \mathrm{x}$ & 0,9966 \\
metribuzin & 9,2 & ECD & $\mathrm{Y}=66890,1+112224,2 \mathrm{x}$ & 0,9941 \\
\hline
\end{tabular}

$\operatorname{tr}=$ tempo de retenção E.Q. = equação da reta $\mathrm{R}=$ coeficiente de correlação
A Tabela 4 apresenta a média do diâmetro de cada amostra antes e após o processo de "clean-up". Para avaliar a possibilidade das amostras serem determinadas sem o processo de "clean-up", as mesmas foram aplicadas diretamente nas cromatoplacas após o procedimento de extração.

Os valores apresentados na Tabela 4 para o cálculo da recuperação dos pesticidas representam um problema sério ocasionado, principalmente, pela imprecisão das medidas. A precisão das réguas comuns é de $\pm 0,5 \mathrm{~mm}$, logo, os erros das medidas estão compreendidos nesta mesma faixa. Ao medir a mancha, por exemplo, com réguas de marcas diferentes obtém-se valores diferentes dos diâmetros da mancha e como os resultados das recuperações são obtidos utilizando-se curvas analíticas (diâmetro da mancha versus quantidade do composto aplicada), um erro na medida de $\pm 0,5 \mathrm{~mm}$ é um erro muito significativo, uma vez que o intervalo das medidas do diâmetro da mancha versus quantidade do composto aplicada é muito pequeno. Isto pode ser melhor visualizado nas curvas analíticas apresentadas na Figura 5. Além disso, é difícil definir exatamente o contorno do diâmetro da mancha.

A Tabela 5 mostra os valores de cada determinação obtidos por GC e HPLC considerando-se 2xLOD e 5xLOD, com a faixa de concentração obtida por TLC.

Os valores de concentração por TLC foram apresentados na tabela por um intervalo de valores. Estes consideram o erro na leitura do diâmetro da mancha que no caso é de $\pm 0,5 \mathrm{~mm}$.

Para a quantificação dos pesticidas estudados em TLC, método de detecção reação de Hill, não é necessário fazer o processo de "clean-up" uma vez que não há interferência do pigmento com o analito após a revelação da placa cromatográfica. Somente o pesticida diuron sofreu interferência com o pigmento, tornando necessário o “clean-up" em coluna de permeação em gel.

Nota-se, também, que por TLC não é possível determinar o valor exato da quantidade de composto, principalmente devido à imprecisão da medida do diâmetro da mancha.

Tabela 4. Média do diâmetro de cada amostra antes e após o processo de "clean-up"

\begin{tabular}{|c|c|c|c|c|c|c|c|c|c|c|c|c|}
\hline \multirow[b]{4}{*}{ PESTICIDA } & \multicolumn{12}{|c|}{ MÉDIA DO DIÂMETRO DA MANCHA } \\
\hline & \multicolumn{6}{|c|}{ com "clean-up" } & \multicolumn{6}{|c|}{ sem "clean-up" } \\
\hline & \multicolumn{3}{|c|}{ Amostras $(1 \mu \mathrm{g})$} & \multicolumn{3}{|c|}{ Amostras $(5 \mu \mathrm{g})$} & \multicolumn{3}{|c|}{ Amostras $(1 \mu \mathrm{g})$} & \multicolumn{3}{|c|}{ Amostras $(5 \mu \mathrm{g})$} \\
\hline & 1 & 2 & 3 & 1 & 2 & 3 & 1 & 2 & 3 & 1 & 2 & 3 \\
\hline atrazina & 5,5 & 6,5 & 4,5 & 7,25 & 7,5 & 7,5 & 5,0 & 4,5 & 4,5 & 6,5 & 6,5 & 6,0 \\
\hline cloroxuron & 4,5 & 5,0 & 5,0 & 4,5 & 5,25 & 6,25 & 5,25 & 6,0 & 6,0 & 7,0 & 7,0 & 7,0 \\
\hline diuron & 5,5 & 6,25 & 6,0 & 7,0 & 6,5 & 7,25 & 9,0 & 9,0 & 8,5 & $*$ & $*$ & $*$ \\
\hline metribuzin & 0 & 0 & 0 & 6,25 & 6,25 & 6,25 & $*$ & $*$ & $*$ & 6,5 & 6,5 & 6,75 \\
\hline
\end{tabular}

* = não detectado

Tabela 5. Comparação dos valores obtidos por GC e HPC considerando-se 2xLOD e 5xLOD, com a faixa de concentração obtida por TLC

\begin{tabular}{|c|c|c|c|c|c|c|c|c|c|}
\hline \multirow{4}{*}{ Pesticida } & \multicolumn{8}{|c|}{ Concentração (ng/mL) } & \multirow{4}{*}{$\begin{array}{c}\mathrm{MRL}^{26} \\
(\mathrm{mg} / \mathrm{kg})\end{array}$} \\
\hline & \multicolumn{6}{|c|}{ com "clean-up" } & \multicolumn{2}{|c|}{ sem "clean-up" } & \\
\hline & \multicolumn{2}{|c|}{ GC } & \multicolumn{2}{|c|}{ HPLC } & \multicolumn{2}{|c|}{ TLC } & \multicolumn{2}{|c|}{ TLC } & \\
\hline & 2xLOD & $5 x L O D$ & 2xLOD & $5 x L O D$ & 2xLOD & $5 x L O D$ & $2 x L O D$ & $5 x L O D$ & \\
\hline atrazina & 0,2 & 0,53 & & & $0,2-0,5$ & $0,7-1,1$ & $0,01-0,3$ & $0,5-0,7$ & \\
\hline cloroxuron & & & 0,21 & 0,79 & $0,1-0,4$ & $0,2-0,7$ & $0,2-0,5$ & $0,4-08$ & \\
\hline diuron & 0,23 & 0,5 & & & $0,2-0,4$ & $0,3-0,5$ & $0,5-0,7$ & & \\
\hline metribuzin & 0,06 & 0,5 & & & $*$ & $0,02-0,03$ & $*$ & $0,2-0,3$ & 0,1 \\
\hline
\end{tabular}

* = não detectado; MRL = limite máximo de resíduo permitido 


\section{CONCLUSÕES}

Pelo método de detecção reação de Hill os herbicidas atrazina, cloroxuron, diuron e metribuzin foram detectados com valores de MDQ entre 0,05 e $1 \mathrm{ng}$ dependendo do composto. O limite de detecção para o pesticida metribuzin utilizando esse método de detecção foi $0,02 \mathrm{ng} / \mu \mathrm{L}$, valor inferior ao limite de resíduo permitido para o tomate que segundo a FAO (Food and Agriculture Organization, é $0,1 \mathrm{mg} / \mathrm{kg}^{26}$. Para esse método de detecção é possível eliminar a etapa de "clean-up" uma vez que não ocorre interferência entre o pigmento e o analito. Assim, há uma diminuição no número de operações manuais que afetam a precisão e exatidão nas análises de resíduos, resultando em considerável economia de reagentes, solventes e tempo de análise.

\section{AGRADECIMENTOS}

Os autores agradecem às agências financiadoras CAPES, CNPq e FAPESP e, também, à Agência Internacional de Energia Atômica pelos pesticidas cedidos. Em especial, SLM agradece à FAPESP pela bolsa de Mestrado concedida.

\section{REFERÊNCIAS}

1. http://www.insite.com.br/bio/wfalmeida/agro1.htm, acessada em Julho 1998.

2. Akerblom, M. Em World Directory of Pesticide Control Organizations Appropriate technology for pesticide analysis in developing countries.; Ekstron, G., ed.; Royal Chemical Society: Nottingan, 1996, p. 19.
3. Ambrus, A.; Development and validation of cost effective screening methods for pesticide residues in vegetables. (Report of technical contract $\mathrm{n}^{\circ}$ 8908) IAEA, 1996.

4. Ambrus, A.; Thier, H.P.; Pure Appl. Chem. 1986, 58, 1035.

5. Cserháti, T.; Forgács, E.; J. Chromatogr. Sci. 1997, 35, 383.

6. Poole, C.F.; Poole, S.K.; J. Chromatogr., A 1995, 703, 573.

7. Sherma, J.; J. Assoc. Anal. Chem. 1999, 82, 48.

8. Breaithwaite, A.; Smith, F.J.; Chromatography Methods; Ed. Blacckie Academic: London, 1996, p. 44.

9. Lehninger, A.L.; Princípios de Bioquímica; Ed. Savier: São Paulo, 1986, p. 436.

10. Kalàsz, H.; Ettre, S.; Báthori, M.; LC-GC 1997, 15, 1044.

11. Rathore, H.S.; J. Chromatogr., A 1997, 733, 5.

12. Mali, B.D.; Garad, M.V.; Patil,V.B.; J. Chromatogr., A 1995, 704, 540.

13. Pasha, A.; Vijayashankar, Y.N.; Analyst 1993, 118, 777.

14. Patil, V.B.; Shingare, M.S.; Analyst 1994, 119, 415.

15. Zoun, P.E.F.; Spierenbur, Th.J.; J. Chromatogr., A 1989, 462, 448.

16. Rathore, H.S.; Begun, T.; J. Chromatogr. 1993, 643, 271.

17. Sundarajan, R.; Chawla, P.; J. Assoc. Off. Anal. 1983, 66, 1009.

18. Patil, V.B.; J; . Chromatogr. 1990, 519, 268.

19. Rathore, H.S.; Begun, T. ; J. Chromatogr. 1993, 643, 321.

20. Patil, V.B.; Sevalkar, M.T.; Palikar, S.V.; Analyst 1992, 117, 75.

21. Tekel, J.; Varverkova, S.; Kovacikova, J.; Holla, M.; Havranek, E.; Pharmacie 1994, 49, 899.

22. http://www.agridata.mg.gov.br/tomate.htm, acessada em Dezembro 1998.

23. Úngaro, M.T.; Pigati, P.; Guindani, C.M.A.; FerreiraA, M.S.; Genebra, A.B.; Ishizaki, T.; O Biológico 1983, 49, 1.

24. Tomlin, C.; The Pesticide Manual: Incorporating the Agrochemicals Handbooks; Ed. The Royal Society of Chemistry: Notthingan, 1995, p. 1341 .

25. Mesquita, T.B.; Riegg, E.F.; Ciência e Cultura 1984, 36, 446.

26. http://apps.fao.org/csv_down, acessada em Dezembro 1998. 